\title{
Galkin Quandles, Pointed Abelian Groups, and Sequence A000712
}

\author{
W. Edwin Clark \\ Department of Mathematics and Statistics \\ University of South Florida \\ Tampa, FL 33620, U.S.A. \\ wclark@mail.usf .edu
}

\author{
Xiang-dong Hou \\ Department of Mathematics and Statistics \\ University of South Florida \\ Tampa, FL 33620, U.S.A. \\ xhou@usf .edu
}

Submitted: Aug 31, 2012; Accepted: Feb 20, 2013; Published: Mar 1, 2013

Mathematics Subject Classifications: 05A17, 20K30, 57M27

\begin{abstract}
For each pointed abelian group $(A, c)$, there is an associated Galkin quandle $G(A, c)$ which is an algebraic structure defined on $\mathbb{Z}_{3} \times A$ that can be used to construct knot invariants. It is known that two finite Galkin quandles are isomorphic if and only if their associated pointed abelian groups are isomorphic. In this paper we classify all finite pointed abelian groups. We show that the number of nonisomorphic pointed abelian groups of order $q^{n}\left(q\right.$ prime) is $\sum_{0 \leqslant m \leqslant n} p(m) p(n-m)$, where $p(m)$ is the number of partitions of integer $m$.
\end{abstract}

Keywords: Galkin quandle; knot; Frobenius symbol; partition number; pointed abelian group

\section{Introduction}

The purpose of this paper is to demonstrate some nice connections between the three objects in the title: Galkin quandle, pointed abelian group, and sequence $A 000712$ (number of partitions of $n$ into parts of 2 kinds). First, let us describe the three objects briefly.

\section{Galkin quandles}

A quandle is a set $X$ equipped with an operation $*$ satisfying the following conditions.

(i) For each $x \in X, x * x=x$.

(ii) For each $y \in X$, the mapping $x \mapsto x * y$ is a permutation of $X$.

(iii) For all $x, y, z \in X,(x * y) * z=(x * z) *(y * z)$. 
A coloring of an oriented knot diagram by a quandle $(X, *)$ is a labeling of the arcs of the diagram by the elements of $X$ such that at each crossing the rule depicted in Figure 1 is observed. The number of colorings of a knot $K$ by a quandle $X$, denoted by $N_{X}(K)$, is an effective knot invariant that can be used to distinguish many nonequivalent knots $[2,3,6]$.

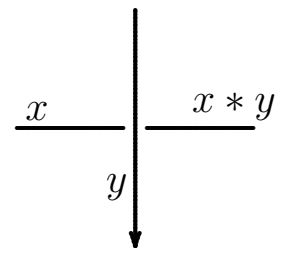

Figure 1: Colors at a crossing

Define $\mu, \tau: \mathbb{Z}_{3} \rightarrow \mathbb{Z}$ by

$$
\mu(x)=\left\{\begin{array}{ll}
2 & \text { if } x=0, \\
-1 & \text { if } x \neq 0,
\end{array} \quad \tau(x)= \begin{cases}1 & \text { if } x=2 \\
0 & \text { if } x \neq 2\end{cases}\right.
$$

Let $A$ be an abelian group and $c \in A$. For $(x, a),(y, b) \in \mathbb{Z}_{3} \times A$, define

$$
(x, a) *(y, b)=(-x-y,-a+\mu(x-y) b+\tau(x-y) c) .
$$

The Galkin quandle, denoted by $G(A, c)$, is the structure $\left(\mathbb{Z}_{3} \times A, *\right)$. This construction was first given by Galkin in [4] for $A=\mathbb{Z}_{p}$ and was recently generalized to any abelian group $A$ in [3]. For more properties of Galkin quandles, see [3].

\section{Pointed abelian groups}

A pointed abelian group is a pair $(A, c)$ where $A$ is an abelian group and $c \in A$. A morphism from a pointed abelian group $(A, c)$ to another pointed abelian group $\left(A^{\prime}, c^{\prime}\right)$ is a homomorphism $f: A \rightarrow A^{\prime}$ such that $f(c)=c^{\prime}$. The category of pointed abelian groups is denoted by $\mathbf{A} \mathbf{b}_{0}$.

\section{Sequence $A 000712$}

In the On-Line Encyclopedia of Integer Sequences [8], $A 000712$ is the sequence $\{a(n)\}_{n=0}^{\infty}$, where $a(n)$ is number of partitions of $n$ into parts of 2 kinds. One has

$$
\sum_{n=0}^{\infty} a(n) x^{n}=\prod_{m=1}^{\infty} \frac{1}{\left(1-x^{m}\right)^{2}}
$$

and

$$
a(n)=\sum_{0 \leqslant m \leqslant n} p(m) p(n-m)
$$


where $p(m)$ is the number of partitions of $m$. For many other interpretations of $a(n)$, see [8].

Let $\mathbf{Q}$ denote the category of quandles, where the morphisms are quandle homomorphisms. For each pointed abelian group $(A, c)$, define $\mathcal{F}(A, c)=G(A, c)$, and for each morphism $f:(A, c) \rightarrow\left(A^{\prime}, c^{\prime}\right)$ of pointed abelian groups, let $\mathcal{F} f: G(A, c) \rightarrow G\left(A^{\prime}, c^{\prime}\right)$ be the quandle homomorphism defined by

$$
(\mathcal{F} f)(x, a)=(x, f(a)), \quad(x, a) \in \mathbb{Z}_{3} \times A .
$$

Then $\mathcal{F}$ is a functor from $\mathbf{A} \mathbf{b}_{0}$ to $\mathbf{Q}$ [3]. In particular, if $(A, c)$ and $\left(A^{\prime}, c^{\prime}\right)$ are isomorphic pointed abelian groups, then $G(A, c)$ and $G\left(A^{\prime}, c^{\prime}\right)$ are isomorphic quandles. For finite Galkin quandles, the converse is also true: If $G(A, c)$ and $G\left(A^{\prime}, c^{\prime}\right)$ are isomorphic quandles, then $(A, c)$ and $\left(A^{\prime}, c^{\prime}\right)$ are isomorphic pointed abelian groups [3]. Therefore, classification of finite Galkin quandles is the same as classification of finite pointed abelian groups.

Let $(A, c)$ be a finite pointed abelian group. We use $q$ to denote a prime to avoid confusion with the partition number $p(n)$. For each prime $q$, denote the $q$-part of $A$ by $A_{q} . \quad\left(A_{q}=\left\{a \in A: q^{n} a=0\right.\right.$ for some $\left.n \geqslant 0\right\}$.) Then $A=\bigoplus_{q} A_{q}$. Write $c=\sum_{q} c_{q}$, $c_{q} \in A_{q}$. Then

$$
(A, c)=\bigoplus_{q}\left(A_{q}, c_{q}\right)
$$

where the meaning of the direct sum of pointed abelian groups is self explaining. If $\left(A^{\prime}, c^{\prime}\right)$ is a another finite pointed abelian group, then $(A, c) \cong\left(A^{\prime}, c^{\prime}\right)$ if and only if $\left(A_{q}, c_{q}\right) \cong\left(A_{q}^{\prime}, c_{q}^{\prime}\right)$ for all primes $q$. Therefore, to classify all finite pointed abelian groups, it suffices to classify all finite pointed abelian $q$-groups.

Let $A$ be a finite abelian $q$-group and let $\operatorname{Aut}(A)$ act on $A$ naturally. For $c, c^{\prime} \in A$, the pointed abelian groups $(A, c)$ and $\left(A, c^{\prime}\right)$ are isomorphic if and only if $c$ and $c^{\prime}$ are in the same $\operatorname{Aut}(A)$-orbit of $A$. The automorphism group $\operatorname{Aut}(A)$ is well known $[5,7]$. In section 2, we describe the orbit representatives of $A$ under the action of $\operatorname{Aut}(A)$. Thus a classification of finite pointed abelian $q$-groups is obtained. This classification allows us to compute the number $N(n)$ of nonisomorphic pointed abelian groups of order $q^{n}$. $(N(n)$ is independent of q.) The initial formula for $N(n)$ resulting from the classification is rather complicated and does not suggest any connection to any well known sequence. However, a search through the On-Line Encyclopedia of Integer Sequences (OEIS) shows that the numerical values of $N(n)$ that we have computed match the sequence $A 000712$. In section 3, we confirm that $N(n)$ is indeed the sequence $A 000712$. The proof is rather tricky; the key step is a formula for the partition number $p(n)$ based on a slight variation of the Frobenius symbol of a partition.

\section{Classification of Finite Pointed Abelian q-Groups}

Let $q$ be a prime and

$$
A=A_{1} \oplus \cdots \oplus A_{k},
$$


where $A_{i}=\mathbb{Z}_{q^{i}}^{n_{i}}, 1 \leqslant e_{1}<\cdots<e_{k}$ and $n_{i}>0,1 \leqslant i \leqslant k$. Let $\pi_{i}: A \rightarrow A_{i}$ be the projection and $\iota_{j}: A_{j} \rightarrow A$ be the inclusion. Then

$$
\operatorname{End}_{\mathbb{Z}}(A)=\bigoplus_{i, j} \iota_{j} \operatorname{Hom}_{\mathbb{Z}}\left(A_{i}, A_{j}\right) \pi_{i}
$$

The mapping $\iota_{j}() \pi_{i}: \operatorname{Hom}_{\mathbb{Z}}\left(A_{i}, A_{j}\right) \rightarrow \iota_{j} \operatorname{Hom}_{\mathbb{Z}}\left(A_{i}, A_{j}\right) \pi_{i}$ is an isomorphism. We will identify $\operatorname{Hom}_{\mathbb{Z}}\left(A_{i}, A_{j}\right)$ with $\iota_{j} \operatorname{Hom}_{\mathbb{Z}}\left(A_{i}, A_{j}\right) \pi_{i}$ through this isomorphism. Thus we can write

$$
\operatorname{End}_{\mathbb{Z}}(A)=\bigoplus_{i, j} \operatorname{Hom}_{\mathbb{Z}}\left(A_{i}, A_{j}\right)
$$

For $\sigma=\sum_{i, j} \sigma_{i j} \in \operatorname{End}_{\mathbb{Z}}(A)$, where $\sigma_{i j} \in \operatorname{Hom}_{\mathbb{Z}}\left(A_{i}, A_{j}\right)$, it is well known that $\sigma \in \operatorname{Aut}(A)$ if and only if $\sigma_{i i} \in \operatorname{Aut}\left(A_{i}\right)$ for all $1 \leqslant i \leqslant k[5,7]$.

Theorem 1. Let $A$ be a finite abelian q-group written in the form (1). For each $1 \leqslant i \leqslant k$, choose $\epsilon_{i} \in A_{i} \backslash q A_{i}$. Let $\mathcal{I}\left(e_{1}, \ldots, e_{k}\right)$ be the set whose elements are sequence of integer pairs $\left(i_{1}, f_{1}\right), \ldots,\left(i_{l}, f_{l}\right)$ satisfying

(i) $l \geqslant 0$, (the sequence is empty when $l=0$,)

(ii) $1 \leqslant i_{1}<\cdots<i_{l} \leqslant k$,

(iii) $0 \leqslant f_{s} \leqslant e_{i_{s}}-1,1 \leqslant s \leqslant l$,

(iv) $0<f_{s+1}-f_{s}<e_{i_{s+1}}-e_{i_{s}}, 1 \leqslant s \leqslant l-1$.

Then as $\left(i_{1}, f_{1}\right), \ldots,\left(i_{l}, f_{l}\right)$ runs through $\mathcal{I}\left(e_{1}, \ldots, e_{k}\right)$,

$$
\sum_{s=1}^{l} q^{f_{s}} \epsilon_{i_{s}}
$$

gives a complete list of orbit representatives of $A$ under the action of $\operatorname{Aut}(A)$.

Proof. $1^{\circ}$ We first show that for each $x \in A$, there exists $\sigma \in \operatorname{Aut}(A)$ such that $\sigma(x)$ is of the form (2).

Clearly, the $\operatorname{Aut}\left(A_{i}\right)$-orbits of $A_{i}$ are represented by 0 and $q^{t} \epsilon_{i}, 0 \leqslant t \leqslant e_{i}-1$. Thus there exists $\sigma \in \operatorname{Aut}(A)$ such that

$$
\sigma(x)=\sum_{1 \leqslant s \leqslant l} q^{f_{s}} \epsilon_{i_{s}}
$$

where $1 \leqslant i_{1}<\cdots<i_{l} \leqslant k$ and $0 \leqslant f_{s} \leqslant e_{i_{s}}-1,1 \leqslant s \leqslant l$. We further assume that $\sigma$ is so chosen that $l$ is minimum. We claim that

$$
0<f_{s+1}-f_{s}<e_{i_{s+1}}-e_{i_{s}}, \quad 1 \leqslant s \leqslant l-1
$$


If, to the contrary of $(3), f_{m+1}-f_{m} \leqslant 0$ for some $1 \leqslant m \leqslant l-1$, then there exists $\alpha \in \operatorname{Hom}_{\mathbb{Z}}\left(A_{i_{m+1}}, A_{i_{m}}\right)$ such that $\alpha\left(q^{f_{m+1}} \epsilon_{i_{m+1}}\right)=q^{f_{m}} \epsilon_{i_{m}}$. Let $\beta=\operatorname{id}_{A}-\alpha \in \operatorname{Aut}(A)$. Then

$$
\beta \sigma(x)=\beta\left(\sum_{1 \leqslant s \leqslant l} q^{f_{s}} \epsilon_{i_{s}}\right)=\sum_{\substack{1 \leqslant s \leqslant l \\ s \neq m}} q^{f_{s}} \epsilon_{i_{s}},
$$

which is a contradiction to the minimality of $l$.

If, to the contrary of (3), $f_{m+1}-f_{m} \geqslant e_{i_{m+1}}-e_{i_{m}}$ for some $1 \leqslant m \leqslant l-1$, then there exists $\alpha^{\prime} \in \operatorname{Hom}_{\mathbb{Z}}\left(A_{i_{m}}, A_{i_{m+1}}\right)$ such that $\alpha^{\prime}\left(q^{f_{m}} \epsilon_{i_{m}}\right)=q^{f_{m+1}} \epsilon_{i_{m+1}}$. Let $\beta^{\prime}=\mathrm{id}_{A}-\alpha^{\prime} \in$ $\operatorname{Aut}(A)$. Then

$$
\beta^{\prime} \sigma(x)=\sum_{\substack{1 \leqslant s \leqslant l \\ s \neq m+1}} q^{f_{s}} \epsilon_{i_{s}}
$$

which is again a contradiction.

So (3) is proved. Now $\sigma(x)$ is of the form (2) and the sequence $\left(i_{1}, f_{1}\right), \ldots,\left(i_{l}, f_{l}\right)$ satisfies conditions (i) - (iv).

$2^{\circ}$ Assume that $\left(i_{1}, f_{1}\right), \ldots,\left(i_{l}, f_{l}\right)$ and $\left(j_{1}, g_{1}\right), \ldots,\left(j_{m}, g_{m}\right)$ are two different sequences in $\mathcal{I}\left(e_{1}, \ldots, e_{k}\right)$. We show that $\sum_{1 \leqslant s \leqslant l} q^{f_{s}} \epsilon_{i_{s}}$ and $\sum_{1 \leqslant s \leqslant m} q^{g_{s}} \epsilon_{j_{s}}$ do not belong to the same $\operatorname{Aut}(A)$-orbit.

Without loss of generality, we may assume that there exists $1 \leqslant u \leqslant l$ such that either $i_{u} \notin\left\{j_{1}, \ldots, j_{m}\right\}$ or $i_{u}=j_{v}$ for some $1 \leqslant v \leqslant m$ but $f_{u}<g_{v}$. For each $\sigma \in \operatorname{Aut}(A)$ written in the form $\sigma=\sum_{i, j} \sigma_{i j}$, where $\sigma_{i j} \in \operatorname{Hom}_{\mathbb{Z}}\left(A_{i}, A_{j}\right), \sigma_{i i} \in \operatorname{Aut}\left(A_{i}\right)$, the $A_{i_{u}}$-component of $\sigma\left(\sum_{1 \leqslant s \leqslant l} q^{f_{s}} \epsilon_{i_{s}}\right)$ is

$$
\sum_{1 \leqslant i \leqslant k} \sum_{1 \leqslant s \leqslant l} \sigma_{i, i_{u}}\left(q^{f_{s}} \epsilon_{i_{s}}\right)
$$

Because of condition (iv), we have

$$
\sigma_{i, i_{u}}\left(q^{f_{s}} \epsilon_{i_{s}}\right) \in \begin{cases}q^{f_{u}} A_{i_{u}} \backslash q^{f_{u}+1} A_{i_{u}} & \text { if } s=u, i=i_{u}, \\ q^{f_{u}+1} A_{i_{u}} & \text { otherwise. }\end{cases}
$$

So the $A_{i_{u}}$-component of $\sigma\left(\sum_{1 \leqslant s \leqslant l} q^{f_{s}} \epsilon_{i_{s}}\right)$ belongs to $q^{f_{u}} A_{i_{u}} \backslash q^{f_{u}+1} A_{i_{u}}$. On the other hand, the $A_{i_{u}}$-component of $\sum_{1 \leqslant s \leqslant m} q^{g_{s}} \epsilon_{j_{s}}$ belongs to $q^{f_{u}+1} A_{i_{u}}$. Thus

$$
\sigma\left(\sum_{1 \leqslant s \leqslant l} q^{f_{s}} \epsilon_{i_{s}}\right) \neq \sum_{1 \leqslant s \leqslant m} q^{g_{s}} \epsilon_{j_{s}}
$$

\section{Number of Nonisomorphic Finite Pointed Abelian $q$-Groups}

It follows from Theorem 1 that the number of nonisomorphic pointed abelian groups with the underlying group $\mathbb{Z}_{q^{e_{1}}}^{n_{1}} \oplus \cdots \oplus \mathbb{Z}_{q^{e_{k}}}^{n_{k}}\left(1 \leqslant e_{1}<\cdots<e_{k}, n_{i}>0\right)$ is $\left|\mathcal{I}\left(e_{1}, \ldots, e_{k}\right)\right|$. 
Consequently, the number of nonisomorphic pointed abelian groups of order $q^{n}$, denoted by $N(n)$, is given by

$$
N(n)=\sum_{\substack{1 \leqslant e_{1}<\cdots<e_{k} \\ n_{1}, \ldots, n_{k}>0 \\ n_{1} e_{1}+\cdots+n_{k} e_{k}=n}}\left|\mathcal{I}\left(e_{1}, \ldots, e_{k}\right)\right| .
$$

The values of $N(0), N(1), \ldots$ are

$$
1,2,5,10,36,65,110,185,300,481, \ldots
$$

which point to the sequence $A 000712$ as a possible match. However, in the form (4), it is not clear that $N(n)$ is the sequence $A 000712$.

Theorem 2. $N(n)$ is the sequence A000712. Namely,

$$
N(n)=\sum_{0 \leqslant m \leqslant n} p(m) p(n-m), \quad n \geqslant 0 .
$$

The key step in the proof of Theorem 2 is the following lemma.

Lemma 3. For integers $k, l \geqslant 0$ define

$$
\begin{aligned}
& A(k, l)=\left\{\left(x_{1}, \ldots, x_{k}\right) \in \mathbb{Z}^{k}: 1 \leqslant x_{1}<\cdots<x_{k}, x_{1}+\cdots+x_{k}=l\right\}, \\
& B(k, l)=\left\{\left(x_{1}, \ldots, x_{k}\right) \in \mathbb{Z}^{k}: 0 \leqslant x_{1}<\cdots<x_{k}, x_{1}+\cdots+x_{k}=l\right\} .
\end{aligned}
$$

Then

$$
p(n)=\sum_{0 \leqslant k \leqslant l \leqslant n}|A(k, l)||B(k, n-l)|=\sum_{0 \leqslant k \leqslant l \leqslant n}|B(k, l-k)||B(k, n-l)| .
$$

Proof. Since $|A(k, l)|=|B(k, l-k)|$, we only have to prove the first equal sign in (6).

In fact, there is a bijection between the set of partitions of $n$ and

$$
\bigcup_{0 \leqslant k \leqslant l \leqslant n} A(k, l) \times B(k, n-l) .
$$

Given $\left(x_{1}, \ldots, x_{k}\right) \in A(k, l)$ and $\left(y_{1}, \ldots, y_{k}\right) \in B(k, n-l)$, we can build a Ferrers diagram herringbone style that corresponds to the a partition of $n$ : We create vertical blocks of sizes $x_{k} \times 1, x_{k-1} \times 1, \ldots, x_{1} \times 1$ and horizontal blocks of sizes $1 \times y_{k}, 1 \times y_{k-1}, \ldots, 1 \times y_{1}$, and we pave these blocks into a Ferrers diagram as shown in Figure 2.

Conversely, given a Ferrers diagram, we can retrieve two sequences $\left(x_{1}, \ldots, x_{k}\right) \in$ $A(k, l)$ and $\left(y_{1}, \ldots, y_{k}\right) \in B(k, n-l)$ in the order of $x_{k}, y_{k}, x_{k-1}, y_{k-1}, \ldots, x_{1}, y_{1}$ as depicted in Figure 2. (Note that it is necessary that $y_{1}$ be allowed to be 0.) Therefore we have the desired bijection. 


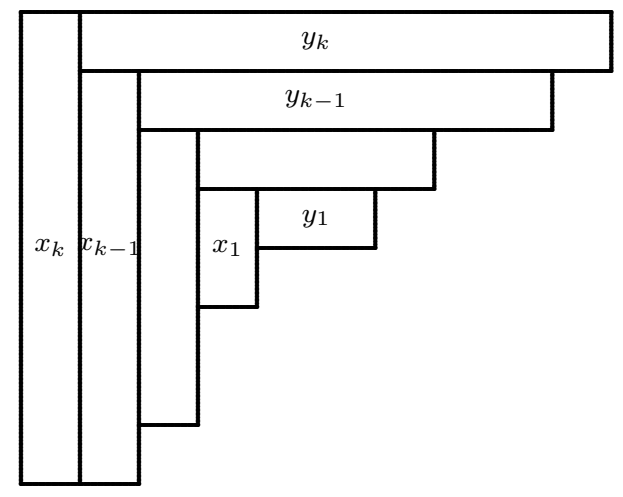

Figure 2: The Ferrers diagram corresponding to $\left(x_{1}, \ldots, x_{k}\right),\left(y_{1}, \ldots, y_{k}\right)$

Remark. The herringbone construction in Figure 2 is a slight variation of the Frobenius symbol of a partition. The array

$$
\left(\begin{array}{cccc}
y_{k} & y_{k-1} & \cdots & y_{1} \\
x_{k}-1 & x_{k-1}-1 & \cdots & x_{1}-1
\end{array}\right)
$$

is the Frobenius symbol of the partition in Figure 2 [1]. Thus Lemma 3 is essentially counting of Frobenius symbols of partitions. For a comprehensive treatise of Frobenius symbols and generalized Frobenius partitions, see [1].

Proof of Theorem 2. Each isomorphic class of pointed abelian groups of order $q^{n}$ is uniquely determined by the following data:

$$
\begin{aligned}
& 1 \leqslant e_{1}<\cdots<e_{k}, n_{1}, \ldots, n_{k}>0 \text { such that } n_{1} e_{1}+\cdots+n_{k} e_{k}=n \\
& \left(\left(f_{1}, i_{1}\right), \ldots,\left(f_{l}, i_{l}\right)\right) \in \mathcal{I}\left(e_{1}, \ldots, e_{k}\right) .
\end{aligned}
$$

Each set of such data can be obtained exactly once through the following steps;

Step 1. Choose $\left(x_{1}, \ldots, x_{l}\right) \in A(l, u),\left(y_{1}, \ldots, y_{l}\right) \in B(l, m-u)$, where $0 \leqslant l \leqslant u \leqslant$ $m \leqslant n$.

Step 2. Choose a partition $\lambda$ of $n-m$. The union of $\lambda$ and $x_{1}+y_{1}, \ldots, x_{l}+y_{l}$ is a partition $\mu$ of $n$. Write

$$
\mu=(\underbrace{e_{1}, \ldots, e_{1}}_{n_{1}}, \ldots, \underbrace{e_{k}, \ldots, e_{k}}_{n_{k}})
$$

where $1 \leqslant e_{1}<\cdots<e_{k}, n_{1}, \ldots, n_{k}>0$.

Step 3. Let $f_{s}=x_{s}, 1 \leqslant s \leqslant l$ and let $i_{s}$ be defined by $x_{s}+y_{s}=e_{i_{s}}$.

To observe how these steps are actually carried out, see Example 4.

For each $0 \leqslant m \leqslant n$, the number of choices in step 1 is

$$
\left.\sum_{0 \leqslant l \leqslant u \leqslant m}|A(l, u)||B(l, m-u)|=p(m) \quad \text { (by Theorem } 3\right) .
$$


The number of choices in step 2 is $p(n-m)$ and the number of choices in step 3 is 1 . Thus we have

$$
N(n)=\sum_{0 \leqslant m \leqslant n} p(m) p(n-m)
$$

Example 4. Assume $n=20$.

Example of step 1. Choose $(1,3,5) \in A(3,9)$ and $(1,2,4) \in B(3,7)$. Note that $m=16$. Example of step 2. $n-m=4$. Choose $\lambda=(1,1,2) \vdash 4$. Then

$$
\begin{aligned}
\mu & =(1,1,2,2,5,9), \\
\left(e_{1}, e_{2}, e_{3}, e_{4}\right) & =(1,2,5,9), \\
\left(n_{1}, n_{2}, n_{3}, n_{4}\right) & =(2,2,1,1) .
\end{aligned}
$$

Step 3. We have $\left(f_{1}, f_{2}, f_{3}\right)=(1,3,5)$. Since $x_{1}+y_{1}=2=e_{2}$, we have $i_{1}=2$. In the same way, $\left(i_{1}, i_{2}, i_{3}\right)=(2,3,4)$.

\section{Acknowledgment}

We thank Professor George E. Andrews for pointing out the connection of Lemma 3 with the Frobenius symbol of a partition.

\section{References}

[1] G. E. Andrews. Generalized Frobenius Partitions. Mem. Amer. Math. Soc., vol. 49, no. 301. AMS, Providence, RI, 1984.

[2] S. Carter, S. Kamada, M. Saito. Surfaces in 4-Space. In Low-Dimensional Topology, III, vol. 142 of Encyclopaedia of Mathematical Sciences, Springer-Verlag, Berlin, 2004.

[3] W. E. Clark, M. Elhamdadi, X. Hou, M. Saito, T. Yeatman. Connected quandles associated with pointed abelian groups. Pacific J. Math, to appear.

[4] V. M. Galkin. Quasigroups. Itogi Nauki i Tekhniki. Ser. Algebra. Topol. Geom., 26 : 3 - 44, VINITI, Moscow, 1988 (Russian). Translated in Journal of Soviet Mathematics, 49(3) : 941-967, 1990.

[5] C. J. Hillar and D. L. Rhea. Automorphisms of finite abelian groups. Amer. Math. Monthly, 114 : 917-923, 2007.

[6] D. Joyce. A classifying invariant of knots, the knot quandle. J. Pure Appl. Algebra, $23: 37-65,1982$.

[7] A. Ranum. The group of classes of congruent matrices with application to the group of isomorphisms of any abelian group. Trans. Amer. Math. Soc., 8 : 71-91, 1907.

[8] The On-Line Encyclopedia of Integer Sequences, http://oeis.org/ 